\title{
Q-switched Erbium-doped Fiber Laser with a Black Phosphorus Saturable Absorber
}

\author{
Nurul Nadia Razak, ${ }^{1}$ Anas Abdul Latiff, ${ }^{* 1,2}$ Zahriladha Zakaria, ${ }^{1}$ and Sulaiman Wadi Harun ${ }^{* 3}$ \\ ${ }^{1}$ Faculty of Electronic and Comp. Eng. (FKEKK), Universiti Teknikal Malaysia Melaka, 76100 Melaka, Malaysia \\ ${ }^{2}$ Photonics Research Centre, University of Malaya,50603 Kuala Lumpur, Malaysia \\ ${ }^{3}$ Department of Electrical Eng., Faculty of Engineering, University of Malaya, 50603 Kuala Lumpur, Malaysia
}

Received May 24, 2017; accepted June 28, 2017; published June 30, 2017

\begin{abstract}
A passively Q-switched Erbium-doped fiber laser (EDFL) is demonstrated using a black phosphorus saturable absorber as the Qswitcher. The laser generates a stable pulse operating at a wavelength of $1562.35 \mathrm{~nm}$ within a pump power region from $7.2 \mathrm{~mW}$ to $42.3 \mathrm{~mW}$. At a $980 \mathrm{~nm}$ pump power of $42.3 \mathrm{~mW}$, the EDFL generates an optical pulse train with a repetition rate of $44.72 \mathrm{kHz}$ and pulse width of $9.8 \mu \mathrm{s}$. The maximum pulse energy of $81.5 \mathrm{~nJ}$ is obtained at a pump power of 35.2 $\mathrm{mW}$. A higher performance Q-switched EDFL is expected to be achieved with the optimization of the saturable absorber and laser cavity.
\end{abstract}

Two-dimensional (2D) layered nanomaterials such as transition-metal dichalcogenides (TMDs) and graphene possess unique planar properties, strong intra-layer covalent bonding and high quantum confinements [1]. These features enable the construction of smaller, efficient and flexible optical devices. For example, graphene has been discovered to have advantages in broad saturable absorption and ultra-fast recovery time [2]. However, this material has its drawbacks such as low absorption efficiency (2.3\%/layer) and absence of a bandgap. TMD materials such as molybdenum disulphide $\left(\mathrm{MoS}_{2}\right)$ and tungsten disulfide $\left(\mathrm{WS}_{2}\right)$ were discovered to be able to overcome the weaknesses of graphene by having unique absorption properties and a layer thickness dependent bandgap. However, these materials are difficult to fabricate since they require a complicated process, thus limiting their potential to become a perfect SA.

The emergence of a new SA material, black phosphorus (BP) has shifted the attention of current research once again as it is found to have the most thermodynamically stable allotrope. Its characteristics are quite similar with those of graphene, having a direct band-gap and being composed of a single element. Despite having an anisotropic structure that requires controlled polarization, BP has many unique electronic properties that may unlock considerable potential of electronic device development [3]. Another important feature is the broad nonlinear optical response, which is the key attribute for infrared optoelectronics application. Therefore, BP is a promising material that may find its importance in Qswitched and mode-locked pulse applications [4]. In 2015, Chen et al. demonstrated the generation of Q-

*E-mail: anasabdullatiff@utem.edu.my and swharun@um.edu.my switched and mode-locked pulse operation employing mechanically exfoliated BP in the C-Band region [5]. Subsequently, in 2016, Mohamad et al. demonstrated a similar usage of BP in the ytterbium fiber laser to generate a mode-locked laser [6].

In this paper, we describe the fabrication of BP as SA by a mechanical exfoliation method. The process was done by peeling raw BPs into a thin layer that may fix onto the end-facet of a fiber ferrule. The BP SA usage was demonstrated by placing it inside the EDFL cavity to generate a Q-switching pulse, which indicates the potential of a BP 2D nanomaterial in the field of laser physics.

The mechanical exfoliation method is free from complicated chemical procedures and expensive instruments, and thus its advantages are simplicity and reliability compared to other techniques. In this work, relatively thin flakes were peeled off from a big block of commercially available BP crystal using a clear scotch tape. Then, we repeatedly pressed the flakes stuck on the scotch tape so that the BP flakes become thin enough to transmit light with high efficiency. Later, a small piece of the tape was cut off and attached to a standard FC/PC fiber ferrule end surface with the help of index matching gel. After connecting the ferrule with another FC/PC fiber ferrule using a standard flange adapter, the all-fiber BP based SA was finally realized.

We verified the quality of the BP tape by taking field emission scanning electron microscopy (FESEM) image. The composition of the transferred layers was then confirmed by energy dispersive x-ray spectroscopy (EDS) on the FESEM image. The presence of a BP material was confirmed by the occurrence of higher peak phosphorus at an energy of $2 \mathrm{keV}$ as shown in Fig. 1a. The inset of Fig. 1a shows the FESEM images, which indicates the existence of uniform layers and confirms the absence of $>1 \mu \mathrm{m}$ aggregates or voids in the composite SA. We also performed Raman spectroscopy measurement on the fabricated BP SA tape sample, and the spectrum is shown in Fig. 1b. The spectrum indicates that the BP sample exhibits three distinct Raman peaks at $360 \mathrm{~cm}^{-1}, 438 \mathrm{~cm}^{-1}$, and $465 \mathrm{~cm}^{-1}$, corresponding to $A_{g}^{1}, B_{2 g}$ and $A_{g}^{2}$ vibration modes of layered $\mathrm{BP}$ [7]. While the $B_{2 g}$ and $A_{g}^{2}$ 
correspond to the in-plane oscillation of phosphorus atoms in BP layer, the $A_{g}^{1}$ mode corresponds to the out-ofplane vibration. The ratio between $A_{g}^{1}$ peak and silicon (Si) level at $520 \mathrm{~cm}^{-1}$ estimates the thickness of BP flake as $\sim 200 \mathrm{~nm}$. The thickness for a single-layer BP is about $0.8 \mathrm{~nm}$ [8], thus the prepared BP based SA is expected to consist of about 250 layers.

(a)

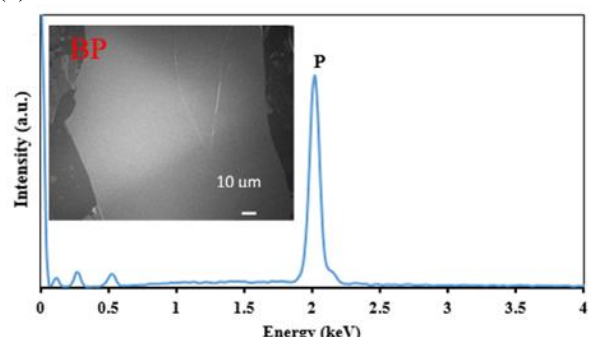

(b)

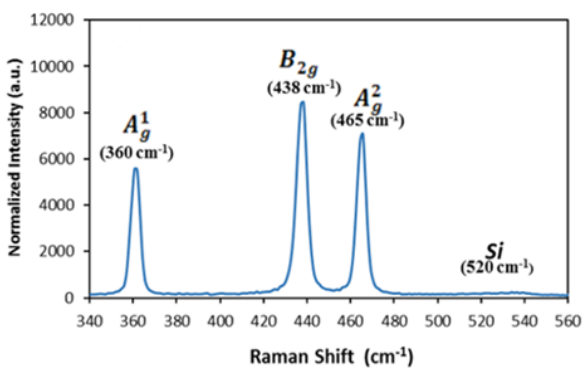

Fig. 1. Characterization of the exfoliated BP layers, transferred onto the tape: (a) EDS data, Inset: FESEM image for BP, (b) Raman spectrum of the BP-SA tape.

Figure 2 shows the experimental setup for the proposed BP based Q-switched EDFL. It uses a 2.8m long Erbiumdoped fiber (EDF) as the gain medium. The EDF was pumped by a $980 \mathrm{~nm}$ laser diode via a 980/1550nm wavelength division multiplexer (WDM). An isolator was spliced after gain medium to prevent backward light operation in the cavity. The BP SA was inserted into a fiber ring laser cavity to produce a passively Q-switched Erbium-doped fiber laser (EDFL). The output from the laser was taken out from the cavity via an optical coupler of $80 / 20$, with the output from $80 \%$ port oscillating inside the cavity while another $20 \%$ port was used to measure the power, spectrum and pulse duration. The results were monitored and analyzed by an optical spectrum analyzer (OSA) with a spectral resolution of $0.02 \mathrm{~nm}, 1.2 \mathrm{GHz}$ InGaAs photodetector combined with $350 \mathrm{MHz}$ oscilloscope and a $7.8 \mathrm{GHz}$ radio frequency spectrum analyzer (RFSA), respectively.

Stable and self-starting Q-switching operation is obtained just by adjusting the pump power over the threshold of $7.2 \mathrm{~mW}$. The Q-switching operation was maintained up to the pump power of $42.3 \mathrm{~mW}$. Low threshold pump obtained from this cavity indicates our proposed cavity has a low intra-cavity loss which contributes towards high optical-to-optical laser efficiency and low power device consumption in future development of a handheld laser.

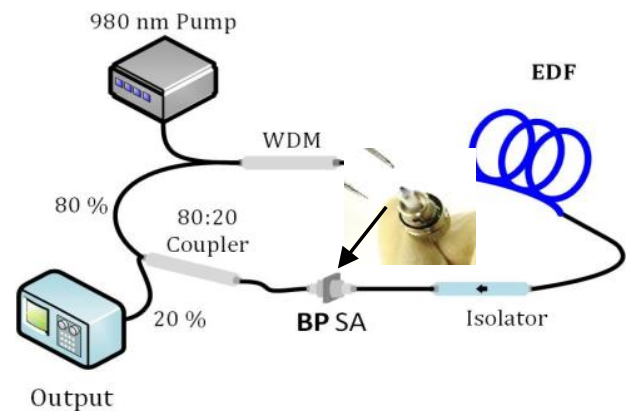

Fig. 2. The schematic setup of the Q-switched EDFL employing the fabricated BP based SA.

Without SA, the continuous-wave lasing started at $6 \mathrm{~mW}$ pump level. Figure 3 shows the output spectrum of the Qswitched laser at a pump power of $35.2 \mathrm{~mW}$. As seen, the laser operates at a wavelength of $1562.35 \mathrm{~nm}$ with a peak of $-7.5 \mathrm{dBm}$ and a $3 \mathrm{~dB}$ spectral bandwidth of $2.4 \mathrm{~nm}$. The pulse broadening is most probably due to the self-phase modulation effect in the ring cavity. Figure 4 shows the oscilloscope traces of a $\mathrm{Q}$-switched pulse train at a pump power of $35.2 \mathrm{~mW}$. It has a pulse-to-pulse separation of $28.5 \mu \mathrm{s}$, corresponding to a pulse repetition rate of $35 \mathrm{kHz}$. As can be seen in the inset image of Fig. 4, a single pulse envelope of the obtained pulse train has a full-wave halfmaximum of $12.8 \mu \mathrm{s}$. There is no distinct amplitude modulation in each envelope of the Q-switched spectrum, which means that the self-mode locking effect on the Qswitching is weak. An additional length of nonlinear fiber in this cavity is expected to induce mode-locking operation. However, more loss was generated and no lasing obtained at the output.

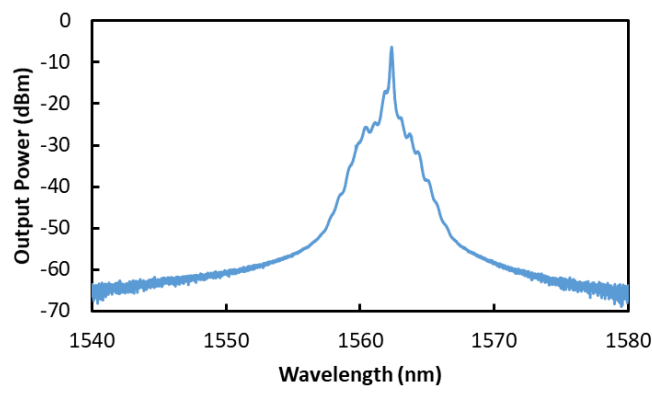

Fig. 3. Output spectrum of the Q-switched EDFL.

Figure 5 shows the repetition rate and the pulse width versus the pump power. The repetition rate of the BP based Q-switched YDFL has a monotonically increasing, near-linear relationship with the pump power level, which is consistent with another Q-switched fiber laser [9, 10]. When the pump power is tuned from 7.2 to $42.3 \mathrm{~mW}$, the pulse train repetition rate varies from 9.61 to $44.72 \mathrm{kHz}$. 


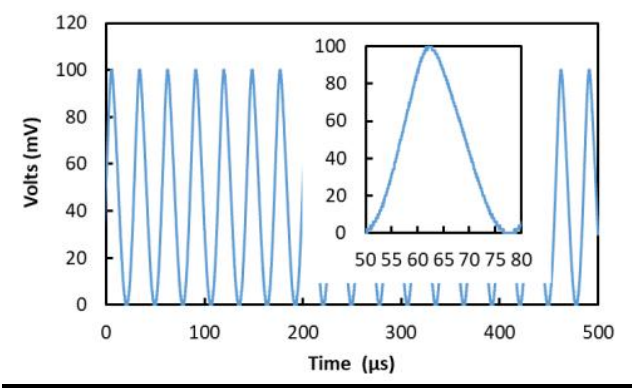

Fig. 4. Typical pulse train for the Q-switched EDFL at a pump power of $35.2 \mathrm{~mW}$. Inset image is a single pulse envelope.

On the other hand, the pulse width is inversely proportional to the pump power, where the pulse duration becomes shorter as the pump power increases. The pulse width monotonically reduces from 40.1 to $9.8 \mu \mathrm{s}$ as the pump power increases from the threshold value to the maximum pump power of $42.3 \mathrm{~mW}$. Figure 6 depicts the average output power and single pulse energy against the pump power. As seen, both output power and pulse energy show an increasing trend with the increase of the pump power. For instance, the output power is linearly increased with a rate of $9.64 \%$ with the pump power. However, we noticed that the pulse energy starts to saturate as the pump power is increased above $35.2 \mathrm{~mW}$. The maximum pulse energy of $81.5 \mathrm{~nJ}$ was obtained at a pump power of $35.2 \mathrm{~mW}$. From the summary of pulsed lasers generated by BP SA, our obtained pulse energy is comparable, and moreover, it has a high repetition rate [11]. The pulse energy could be improved by reducing the insertion loss of the SA and optimizing the laser cavity.

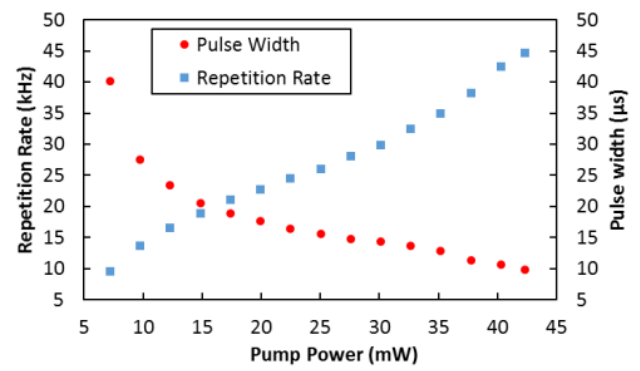

Fig. 5. Repetition rate and pulse width as a function of pump power.

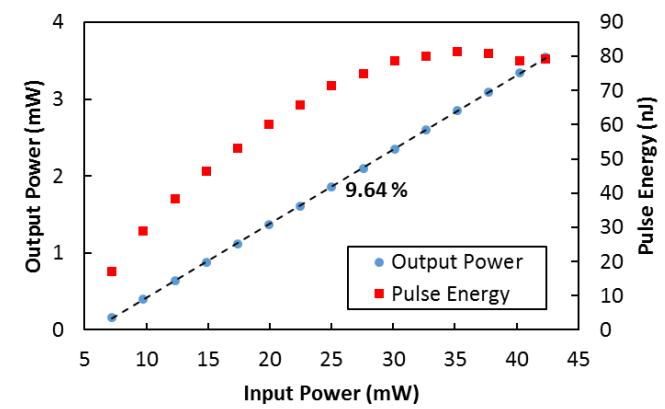

Fig. 6. The output power and calculated pulse energy against the input pump power.
The RF spectrum of the Q-switched pulses is also measured at a pump power of $35.2 \mathrm{~mW}$, and the result is shown in Fig. 7. Its fundamental mode peak locates at the frequency of $35 \mathrm{kHz}$ and has a signal-to-noise ratio (SNR) of $67 \mathrm{~dB}$, which confirms the stability of the pulse. So far, the SA function in BP tape has remained robust after we increased pump power to a maximum limit of $150 \mathrm{~mW}$.

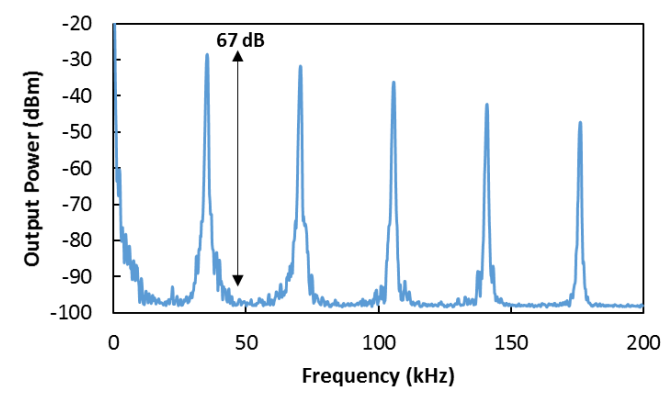

Fig. 7. RF spectrum of the Q-switched pulse train at a pump power of $35.2 \mathrm{~mW}$.

In conclusion, a Q-switched EDFL operating at $1562.35 \mathrm{~nm}$ is successfully demonstrated based a mechanically exfoliated BP as a Q-switcher. When the pump power is tuned from the threshold pump power of 7.2 to $42.3 \mathrm{~mW}$, the pulse train repetition rate varies from 9.61 to $44.72 \mathrm{kHz}$. On the other hand, the pulse width reduces from $40.1 \mu$ s to $9.8 \mu$ s as the pump power increases from the threshold value to the maximum pump power of $42.3 \mathrm{~mW}$. The maximum pulse energy of $81.5 \mathrm{~nJ}$ is obtained at a pump power of $35.2 \mathrm{~mW}$. This result shows the great potential of a BP nanomaterial for application in Q-switching pulse generation

N. N. Razak wishes to thank Ministry of Higher Education, Malaysia for awarding MyPhD Scholarship for her $\mathrm{PhD}$.

\section{References}

[1] R. Mas-Balleste, C. Gomez-Navarro, J. Gomez-Herrero, F. Zamora, Nanoscale 3, 20 (2011).

[2] Z. Sun, T. Hasan, F. Torrisi, D. Popa, G. Privitera, F. Wang, F. Bonaccorso, D.M. Basko, A.C. Ferrari, ACS Nano 4, 803 (2010).

[3] F. Xia, H. Wang, Y. Jia, Nat. Commun. 5 (2014).

[4] G. Sobon, Photon. Research 3, A56 (2015).

[5] Y. Chen, G. Jiang, S. Chen, Z. Guo, X. Yu, C. Zhao, H. Zhang, Q. Bao, S. Wen and D. Tang, Opt. Expr. 23, 12823 (2015).

[6] M. Hisyam, M. Rusdi, A. Latiff, S. Harun, IEEE J. Selected Topics in Quant. Electr. 23, 39 (2017).

[7] S. Sugai, I. Shirotani, Solid State Comm. 53, 753 (1985).

[8] Z.-C. Luo, M. Liu, Z.-N. Guo, X.-F. Jiang, A.-P. Luo, C.-J. Zhao, X.-F. Yu, W.-C. Xu, H. Zhang, Opt. Expr. 23, 20030 (2015).

[9] A. Latiff, M. Rusdi, M. Hisyam, H. Ahmad, S. Harun, J. Modern Opt. 64, 187 (2017)

[10] J. Bogusławski, G. Soboń, K. Tarnowski, R. Zybała, K. Mars, A. Mikuła, K.M. Abramski, J. Sotor, Opt. Eng. 55, 081316 (2016).

[11] Z. Wang, Y. Xu, S. C. Dhanabalan, J. Sophia, C. Zhao, C. Xu, Y. Xiang, J. Li, H. Zhang, IEEE Photonics Journal 8, 1 (2016). 